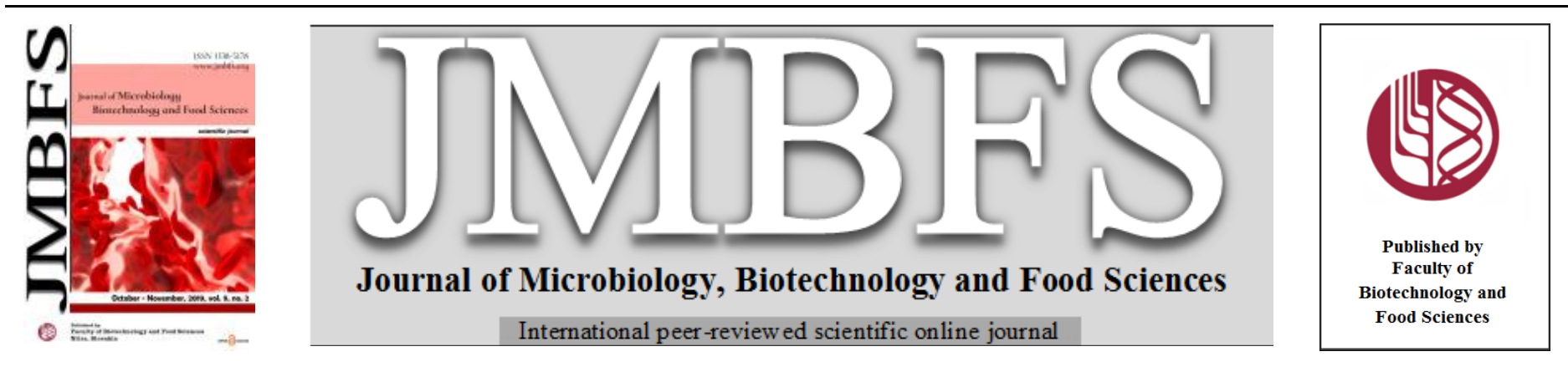

\title{
MEDICINAL ALTERNATIVE FOR CHIKUNGUNYA CURE: A HERBAL APPROACH
}

\author{
Komal Arora, Pushpa C. Tomar*, Pritika Kumari, Anju Kumari
}

Address(es):

Department of Biotechnology Engineering, Faculty of Engineering \& Technology, Manav Rachna International Institute of Research and Studies, Faridabad, Haryana, (INDIA) -121004.

*Corresponding author: tomarpushpa@ rediffmail.com , pushpa.fet@mriu.edu.in

doi: 10.15414/jmbfs.2020.9.5.970-978

\section{ARTICLE INFO}

Received 23. 7. 2019

Revised 16. 11. 2019

Accepted 19. 11. 2019

Published 1. 4. 2020

\section{Review}

OPEN OACCESS $_{\text {A }}$

\begin{abstract}
Recently a major outbreak of chikungunya affected several countries of the world. In India only 62,628 and 64,057 clinically suspect chikungunya cases in 2017 and 2016 respectively and about 9,175 suspected chikungunya cases through April 2018. A self-remitting febrile viral illness, chikungunya fever caused/spread by Aedes aegypti and Aedes albopictus. The name has been originating from a verb in the Kimakonde language, meaning 'to become contorted' means 'stooped' appearance of joint, signalized by fever and joint pain which can sustain for months or even years in some of the patients. Although many medications are available in the market but the effective treatment against the viral infection is still restricted, due to drug resistance, side effects and toxicity. Focusing the problem, this paper mainly concerns on the cause, transmission of chik virus, and how herbal plants, their active constituents and specific medicinal properties used for relieving symptoms and for cure- providing an alternative way for treating chikungunya.
\end{abstract}

\section{INTRODUCTION}

An intense febrile illness- chikungunya caused by chikungunya virus i.e. arthropod-borne alphavirus (Staples et al., 2009). The word chikungunya means "bends up" (Cavrini $\boldsymbol{e t}$ al., 2009) and is characterized by arthritis (joint pain) and rash (Galán-Huerta et al., 2015). It is transmitted by Aedes mosquitoes to humans (da Cunha and Trinta, 2017). Self-bounded infection is observed and severe symptoms usually not last for more than 1-2 weeks but this fever is however, can recurrent in $30-40 \%$ of infected individuals and may continue for years. Chikungunya virus includes under the family of Togaviridae and genus alpha virus (da Cunha and Trinta, 2017). It was first isolated from febrile patient of Tanzania in 1955 (Rahman et al., 2017). The disease fall into three phases: Day 1-21 known as acute phase, Day21-90 known as sub-acute phase and from $90^{\text {th }}$ day onwards called chronic phase (Amin et al., 2017). Chikungunya infection not only causes pain, but also mental health, sleeping disorders and mood swings is observed in patients suffering with chikungunya (Schilte $\boldsymbol{e t}$ al. 2013; Ramachandran et al., 2014)

1. Pathophysiology

1.1 Transmission

Two different cycles are there through which chikungunya virus is transmitted: urban and sylvatic (Ganesan et al., 2017). When transmission is from human to mosquito to human is known as urban cycle whereas when transmission is from animal to mosquito to human is known as sylvatic cycle (Singh and Unni, 2011).

1.2 Target cells

It has been studied from several publications about the sensitivity of different cells to chikungunya virus replication and it has been observed from a series of immortalised primary human cells that epithelial and endothelial cells, primary fibroblasts, monocyte derived macrophages were susceptible to chikungunya virus (Thiberville et al., 2013).

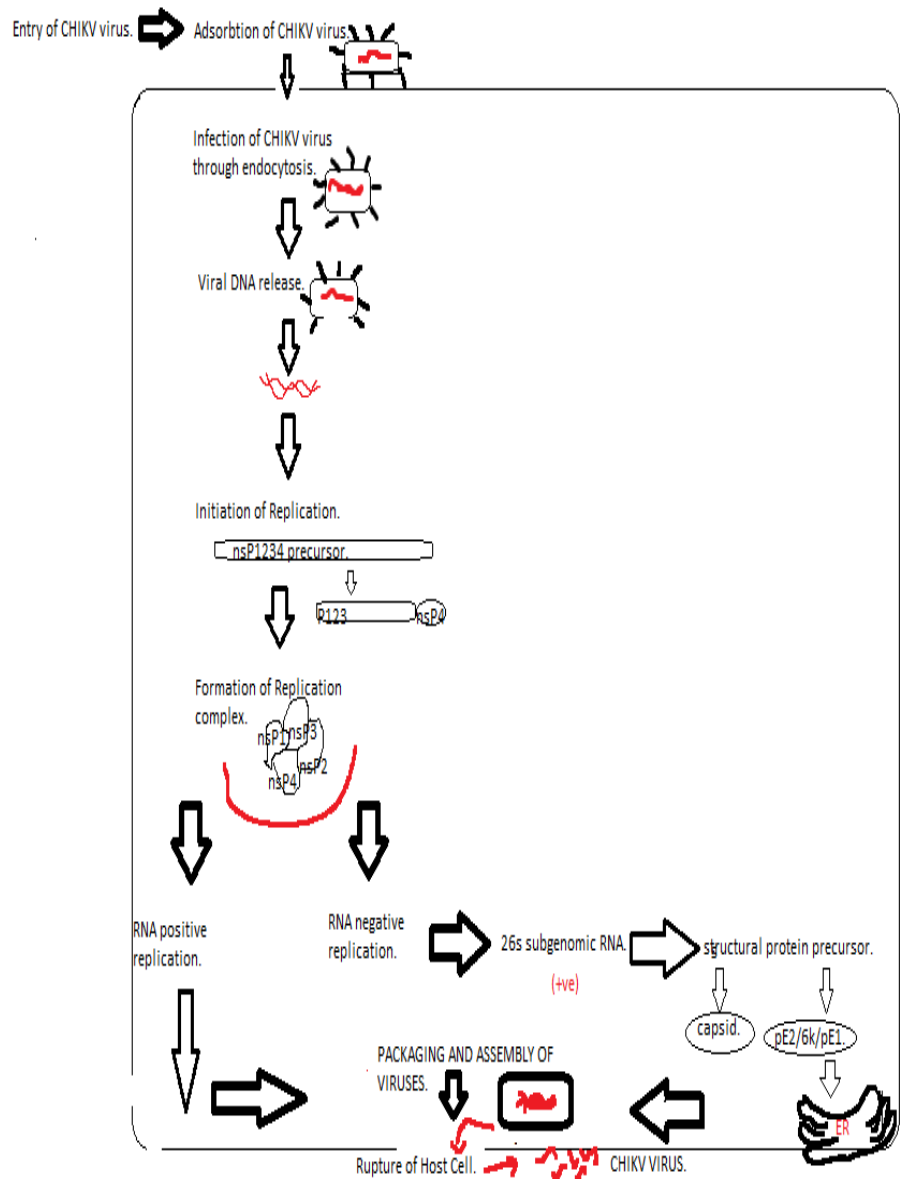

Figure 1 Mechanism of infection of Chikungunya virus 
Entry of chikungunya virus into mammalian epithelial cells occurs via a path of clathrin -independent, Esp-15-dependent and dynamic 2-dependent routes. These all pathways need endocytic signaling combined with some other unknown pathways (Teo et al., 2013).

Due to the capability of chikungunya virus to undergo genetic drifts, it has been proposed that this virus can acquire alternate entry mechanism (Bernard et al., 2010)

\section{Etiology}

A positive-sense single-stranded RNA chikungunya virus consists of 11.8 kilo base pairs along with a capsid and a phospholipid envelope (Strauss and Strauss, 1994), and its genome consists of two open reading frames: the 5ORF, that is translated from genomic RNA and encodes nonstructural proteins such as nsP1,nsP2,nsP3 and nsP4, and 3ORF, that is translated from sub genomic RNA and encodes structural proteins such as capsid(C), envelope(E1 and E2), and two peptides(E3 and 6k) (Schwartz and Albert, 2010). Chikungunya virus includes under the family of Togaviridae and genus alpha virus (Schwartz and Albert, 2010). With the help of phylogenetic analysis, partial sequences of NS4 and E1 genes, three different groups have been found that includes the West African, the East-Central-South African, and the Asian (Strauss and Strauss, 1994; Mohan et al., 2010; Chhabra et al., 2008).

\section{Symptoms of chikungunya}

Symptoms of chikungunya in patient generally arise after the bite of an infected mosquito. Headache, muscle pain, chills, back pain, joint swelling, myalgia, arthralgia, nausea, vomiting, rash, fever and joint pain are some of the common symptoms observed in chikungunya (Rahman et al., 2017).

3.1 Symptoms observed in different phases

Acute phase - high fever, headache, chills, nausea, vomiting, fatigue, back pain, myalgia and arthralgia, along with collateral abnormalities such as anemia, pronounced lymphopenia and/or moderate thrombocytopenia and leucopenia have also been diagnosed (Sharma and Jain, 2018). High levels of liver enzymes, creatinine, creatinine kinase and hypocalcaemia have been also been observed in this phase (Thiberville et al., 2013). Chronic phase- severity of joint pain and presence of swelling in the joints (inflammation) (Sharma and Jain, 2018).

\section{Treatment}

Several medications such as ribavirin, interferon alpha, chloroquine, arbidol, favipiravir, and furin inhibitors are available and have recognized effect against chikungunya virus, although no specific antiviral treatment against infection is available (da Cunha and Trinta, 2017), henceforth there is an immediate need of finding an alternative approach for the treatment of chikungunya by means of herbal ways.

Different plants available for cure and prevention of chikungunya are listed below:

\section{Tinospora cordifolia}

It is also known as guduci in Sanskrit language is a member of family Menispermaceae. Alkaloids, steroids, sesquiterpenoid, phenolics, aliphatic compounds, polysaccharides, diterpenoid lactones, and glycosides are some of the active constituents found in the plant (Ghosh and Saha, 2012). Medicinal properties like anti-diabetic, anti-periodic, anti-spasmodic, anti-inflammatory, anti-arthritic, anti-oxidant, anti-allergic, anti-stress, anti-leprotic, anti-malarial, hepatoprotective, anti-pyretic, anti-microbial, anti-osteoporotic, immunomodulatory and anti-neoplastic activities have been reported in different studies (Ghosh and Saha, 2012). Anti-inflammatory (minimized inflammation in eyes), anti-arthritic (reduces joint pain), immunomodulatory (boosts immune system), anti-allergic (treatment of rashes), anti-pyretic (treatment of fever) are some of the specific medicinal properties that allows the prevention and treatment of chikungunya.

\section{Zingiber officinale}

It belongs to the family Zingiberaceae and is known to be the most important plant that has various nutritional, medicinal properties (Dhanik et al., 2017). It has been reported that essential oils, phenolic compounds, flavonoids, carbohydrates, proteins, alkaloids, lycosides, saponins, steroids, terpenoids, and tannin are the major active constituents present as phytochemical groups (Dhanik et al., 2017). Its pharmacological significance includes its value in the treatment of diabetes, obesity, diarrhea, allergies, pain, fever, rheumatoid arthritis, inflammation, various forms of cancer and thus has various anti-oxidant, anti-microbial, anti-diabetic, anti-cancer, anti-inflammatory, analgesic, antipyretic, immunomodulatory, anti-platelet aggregation, anti-angiogenic, hepatoprotective, larvicidal, anti-emetic, anti-obesity, anti-atherosclerotic, renoprotective, neuroprotective activities (Dhanik et al., 2017). Anti-pyretic, anti-arthritic, analgesics, immunomodulatory, anti-allergic are some of the specific properties that alleviate the symptoms of chikungunya.

\section{Andrographis paniculata}

It is known as King of Bitters whose genus Androraphis belongs to family Acanthaceae (Okhuarobo et $\boldsymbol{a l}$., 2014). Active constituents include diterpenoids, diterpene glycosides, lactones, flavanoids, and flavanoid glycosides, quinic acids, xanthones (Hossain et al., 2014). Biological activities such as anti-viral, antibacterial, immunostimulatory, anti-inflammatory, anti-tumor, anti-diabetic, antimalarial, hepatoprotective, anti-allergic, anti-HIV, anti-platelet aggregation, antioxidant, anti-pyretic, analgesic, larvicidal, renoprotective, anti-fertility, antihyperglycemic, hypolipidemic, cardiovascular, anti-diarrheal effects (Jayakumar et al., 2013). Properties like anti-viral, anti-inflammatory, antiallergic, anti-pyretic, analgesic, anti-diarrheal, immune-stimulatory plays a significant role in reducing symptoms caused by chikungunya.

\section{Ocimum sanctum}

Ocimum known as Tulsi or Holy Basil is a member of family Lamiaceae. It grows as weed and also cultivated in tropical areas (Buddhadev et al., 2014). It consists of complex chemical composition (many nutrients and biologically active compounds) which includes eugenol, urosolic acid, carvacrol, linalool, caryophylline, estragol, rosmarinic acid, apigenin, cirsimaritin, fatty acids, sitosterol, sugars (xylose and polysaccharides), anthocyans (Rahman et al., 2011). It has various pharmacological activities that has the ability to treat many diseases and these activities are anti-microbial, anti-cancer, anti-fungal, immunomodulatory, hepatoprotective, anti-viral, wound healing, anti-pyretic, anti-cataract, anti-hyperlipidemic, anti-coagulant, anti-stress, anti-helminthic, radio protectant, anti-arthritic, anti-diabetic, anti-inflammatory, anti-oxidant, antibiotic, anti-diarrheal, larvicidal, anti-genotoxic, neuro and cardio protective, analgesic (Rahman et al., 2011; Bano et al., 2017). Properties possess by this plant such as anti-pyretic, anti-viral, anti-inflammatory, anti-arthritic, antidiarrheal helps in relieving the symptoms of chikungunya.

\section{Terminalia chebula}

This plant is a member of family Combretaceae-Indian almond family and its phytoconstituents include anthocyanins, alkaloids, tannins (gallic acid, chebulagic acid, punicalagin, chebulanin., corilagin, neochebulinic acid, 1,2,3,4,6-penta-O-galloyl-beta-d-glucose, 1,6-di-o-galloyl-D-glucose, casuarinin, 3,4,6-tri-o-gloooyl-D-lucose, terchebulin), phenolics (chebulunic acid, ellagic acid and anthraquinones), polyphenols(corilagin, galloyl glucose, punicalagin, terflavin A, maslinic acid), fructose, amino acids, succinic acid, betasitosterol, resin, flavanol, glycosides, triterpenoids (Bag et al., 2013).

High medicinal value and pharmacological activity is possesed by this plant for various ailments treatment. This includes anti-oxidant, anti-microbial, antidiabetic, hepatoprotective, anti-inflammatory, anti-mutagenic, anti-proliferative, radioprotective, cardioprotective, anti- arthritic, gastrointestinal motility, wound healing, analgesic, anti-allergic, anti-carcinogenic, cytroprotective renoprotective, anti-viral, adaptogenic and anti-anaphylactic, anti-spasmodic, anti-ulcerogenic, hypolipidemic and hypocholesterolemic, purgative property and immunomodulatory activity (Bag et al., 2013). Significant potential of Terminalia chebula which includes anti-inflammatory, anti-arthritic, analgesic, anti-allergic, anti-viral, immunomodulatory abate the symptoms of chikungunya.

\section{Emblica officinalis (Phyllanica emblica)}

It is generally known as Indian gooseberry or Amla, belongs to the family Euphorbiaceae (Vimala et al., 2011). Higher amount of polyphenols (gallic acid, ellagic acid, different tannins, minerals, vitamins, amino acids, fixed oil) flavonoids(rutin and quercetin), glycosides are the phytoconstituents found in this plant (Variya et al., 2011). Potential of pharmaceutical activities includes antimicrobial, anti-inflammatory, analgesic, anti-pyretic, adaptogenic, hepatoprotective, anti-tumor, anti-ulcerogenic, immunomodulatory, cytroprotective, anti-tussive, radioprotective, anti-cancer, anti-diabetic, antidiarrheal (Gaire and Subedi, 2014; Jain et al., 2015).

\section{Rubia cordifolia}

A perennial, herbaceous climbing plant is belonging to the family Rubiaceae (Kannan, a et al., 2009), often known as Common Madder or Indian Madder (Verma et al., 2016). Active constituents from different parts reported in several studies include anthraquinones and teir glycosides, terpenes, bicyclic hexapeptides, iridoids, carboxylic acids and saccharides (Devi Priya and Siril, 2014). Various pharmaceutical uses have been reported previously which include anti-acne property, anti-arthritic effects, anti-cancer property, anti-inflammation activity, wound healing effects, anti-microbial activity, anti-convulsant activity, anti-diabetic activity, anti-oxidant activity, anti-peroxidative activity, diuretic effects, anti-viral activity, anti-ulcer activity, anti-stress activity, anti-proliferative 
activity, immunomodulatory activity, hepato, neuro and gastroprotective activity, radioprotective (Devi and Siril, 2014). Anti-arthritic, anti-inflammation, antiviral, immunomodulatory activity possessed by tis plant help in soothing the symptoms of chikungunya.

\section{Cyperus rotundus}

Common name of Cyperus rotundus is Nagarmota, belongs to the family Cyperacea (Imam et al., 2014). Chemical components are essential oils, flavanoids, terpenoids, mono-sesquiterpenes, cyprotene, acopaene, cyperene, aselinene, rotundene, valencene, cyperol, gurjunene, trans-calamenene, dcadinene, gcalacorene, cadalene, amuurolene,gmuurolene, cyperotundone, mustakone, isocyperol, acyperone, 4,11-selinnadien-3-one and 1,8-cineole (Imam et al., 2014). Studies based on phytochemical and pharmacological constituents suggests the potential activities of Cyperus rotundus such as antiandrogenic,anti-malarial, anti-uropathogenic, cardioprotective, anti-bacterial, anti-mutagenic,anti-oxidant, anti-inflammatory, anti-diarrheal, anti-genotoxic, anti-convulsant, anti-diabetic, anti-obesity, hepato and neuroprotective activities, anti-atherosclerosis, anti- pyretic, analgesic (Peerzada et al., 2015)

\section{Picrorhiza kurroa}

An important medicinal plant with common name Kutki is a member of family Scrophulariaceae (Masood et al., 2015). Chemical constituents found from different parts of plant are iridoids, acetophenones, cucurbitacins, picroside-I and II (major bioactive compounds), pikuroside, veronicoside, phenol glycosides, curcurbitacin glycosides and 4-hydroxy-3methoxy-acetophenone (Sah and Varshney, 2013). Liver disorders, fever, asthma, jaundice, gastrointestinal and urinary disorders can be treat by this plant and shows activities like antiinflammatory, anti-allergic, immunomodulatory, anti-anaphylactic, anti-hepatitis B surface antigen activity (Sah and Varshney, 2013), anti-microbial, hepatoprotective, anti-oxidant, anti-ulcer, anti-cytochrome action, neuromuscular action, anti-leishmanial, hypolipemic effect, cardioprotective, anti-diabetic, antimalarial activity (Kumar et al., 2013), anti-viral, anti-cancer, anti-cholestatic, anti-asthamatic, analgesic, nephroprotective (Salma et al., 2017).

\section{Pluchea lanceolata}

The genus Pluchea belongs to the family Asteraceae, commony known as Rasna Phytoconstituents present in this plant includes alkaloids, flavonoids, tannins, steroids, glycosides, saponins, terpenoids, proteins, carbohydrates, phenols, lactones (Arya and Patni, 2013).

It shows anti-inflammatory, analgesic, anti-arthritic, anti-pyretic, laxative, thermogenic activities and is used in the treatment of cough, piles, neurological diseases, edema (Srivastava and Shanker, 2012).

\section{Terminalia bellerica}

Commonly known as Baheda, is a member of family Combretaceae (Deb et al., 2016). Phytoconstituents found in this plant includes glucoside, tannins, gallic acid, ethyl gallate, chebulinic acid, lignans (termilignan and thannilignan), $7-$ hydroxy 3' 4' (methylenedioxy) flavone and anolignan B , ellagic acid, galloyl glucose, phyllemblin, beta-sitosterol, mannitol, glucose, fructose, rhamnose (Deb et al., 2016), alkaloids, steroids, terpenoids, saponin (Kadian et al., 2014) Different phytoconstituents present in plant contributed towards many pharmacological activities and are analgesic, anti-diarrhoel, anti-oxidant, antihypertensive, anti-salmonella, anti-spasmodic, anti-microbial, anti-biofilm, anticancer, immunological, wound healing, anti-ulcer, anti-pyretic, anti-mutagenic, thrombolytic, beta lactamase inhibitor activity (Deb et al., 2016) immunomodulatory, anti-biofilm, anti-depressant, anti-diabetic,anti-fungal, antihelminthic, anti-inflammatory, anti-oxidant, , glucoamylse activity, hepatoprotective activity, anti-thrombotic activity (Kadian et al., 2014).

\section{Commiphora wightii}

Common name for Commiphora wightii is guggul, belongs to the family Burseraceae (Sarup et al., 2015). Several phytohconstituents that are responsible for the treatment of variety of diseases are terpenoids (monoterpenoids, sesquiterpenoids, triterpenoids), steroids, flavonoids, guggultetrols, lignans, sugars, amino acids. Another phtoconstituents may include long chain aliphatic tetrols, aliphatic esters, ferulates, carbohydrates, inorganic ions (Sarup et al., 2015). Activities like Anti-inflammatory, anti-obesity (Cunningham et al. 2018), anti-arthritic, anti- viral, anti-microbial, wound healing, anti-tumor, antifertility, cytotoxic activity, cardioprotective, anti-atherosclerotic, anti-oxidant, thyroid stimulatory activity, anti-platelet aggregation, hypolipidemic activity, anti-hyperglycemic are used for the treatment of various ailments such as inflammation, obesity, rheumatism, infection, defects in lipid metabolism (Sarup et al., 2015).

\section{Curcuma longa}

Turmeric's active component, curcumin, belongs to the ginger (Zingiberaceae) family (Kocaadam and Çanlier, 2017; Khajehdehi et al., 2012). Around 235 compounds have been identified; some of them are phenolic compounds, terpenoids, diarylheptanoids (curcuminoids), diarylpentanoids, monoterpenes, sesquiterpwnws, diterpenes, triterpenes, alkaloid, and sterols, etc. (Li et al., 2011). Pharmaceutical significance of Curcuma longa includes anti-inflammatory activity, anti-oxidant, anti-tumor, anti-HIV, anti-protozoal activity, nematocidal activity, anti-venom activity (Araújo et al., 2001), anti-viral activity, antiinfectious activity, wound healing (Joe $\boldsymbol{e t}$ al., 2004), anti-bacterial activity, antifungal activity, anti-mycobacterial activity (Çıkrıkçı et al., 2008), anti-platelet activity, hepatoprotective activity, anti-arthritic activity, anti-proliferative activity, analgesic, anti-malarial activity (Perrone et al., 2015).

\section{Boswellia serrata}

It is a member of family Burseraceae with the common name Indian frankincense (Arora et al., 2013). Chemical constituents found in studies include monoterpenes, diterpenes, triterpenes, pentacyclic triterpenic acids, tetracyclic triterpenic acids (Siddiqui et al., 2011). It exhibits several biological activities such as anti-arthritic, anti-diarrheal, anti-pyretic, anti-inflammatory, antihyperlipidemic, anti-atherosclerotic, analgesic, hepatoprotective, anti-oxidant, diuretic effects, anti-coagulant, anti-tumor, aanti-allergic (skin disorders), cardio and gastroprotective (Siddiqui et al., 2011; Harrasi et al., 2018).

\section{Vitex negundo}

It is commonly known as Nirgundi, belonging to the Verbenaceae family, found mostly in moist areas (Venkateshwarlu, 2012). Main compounds present in this plant are viridiflorol, beta-caryophyllene, sabinene, 4-terpineol, gammaterpinene, caryophyllene oxide, 1-ocetan-3-ol, globulol, laonoid glycosides, negundoside, agnuside, vitegnoside, p-hydroxybenzoic acid, beta-sitostero (Singh et al., 2003; Meena et al., 2011). Pharmaceutical potential and biological activities includes anti-arthriic, anti-inflammatory, analgesic, antihyperpigmentation, immuno-stimulant, hepatoprotective, CNS activity, antiandrogenic activity, enzyme inhibition, mosquito repellant, anti-convulsant, antioxidant, insecticidal and pesticidal activity, anti-hyperglycemic, anti-bacterial, anti-asthmatic, anti-implantation (Venkteshwarlu, 2012; Meena et al., 2011).

\section{Withania somnifera}

This plant of family Solanaceae is commonly known as Ashwagandha (Gupta and Rana, 2007). Phytoconstituents present are alkaloids, steroidal compounds, ergostane type typesteroidllactones, withaferin A, withanolides A-y, withasomniferin-A, withasomidienone, withasomniferols A-C, withanone etc. Additional constituents include saponins, withanolides, withaniol, acylsteryl glucosides, starch, reducing sugar, hantreacetone, ducitol, amino acids. Pharmacological actions of this plant is anti-oxidant effect, chronic stress, nootropic effect, anti-parkinsonian properties, anti-venom, immunomodulation, anti-inflammatory activity, hematopoiesis, anti-tumor activity, hypolipidemic effects, anti-bacterial property, hypothyroid activity, cardioprotective effects, anti-arthritic, anti-asthamatic, spermatoenic activity, neuroprotective activity, hepatoprotective, anti-depresssion and anti-anxiety activity, anti-microbia activity, anti-platelet, anti-coagulant, anti-leishmanial activity, hypoglycaemic, anti-viral, anti-fungal, anti-convulsant, nephroprotective activity (Gupta and Rana, 2007; Singh et al., 2010; Kumar et al., 2015 ).

\section{Glycyrrhiza glabra}

Also known as mullaiti or licorice is a member of family Fabaceae (Nesar et al. 2016). Glycyrrhizin, glycyrrhizinic acid, glabrin A and B, glycyrrhetol, glabrolide, isoglabrolide, isoflavones, coumarins, triterpene sterols, starches, pectins, polysaccharides, simple sugars, gums, mucilage, amino acids, flavonoids, mineral salts, bitters, essential oil, fat, estrogen, tannins, glycosides, protein, resins, volatile oils are some of the phytochemicals present (Nesar et al., 2016; Damle, 2014). Biological activities include anti-inflammatory, antioxidant, anti-ulcer, thrombin inhibitor, anti-bacterial, anti-fungal, anti-tussive, anti-diabetic, anti-cancer, hepatoprotective (Nesar et al., 2016), skin lightening and skin tightening activity, anti-viral effects, anti-coagulant, immunostimulatory activity, anti-hyperglycemic effects, memory boosting activity, hair growth stimulatory activity, anti-bacterial, anti-malarial (Damle, 2014)

\section{Lepidium sativum}

This plant of family Brassicaceae has a common name of Garden cress (Falana et al., 2014). It consists of alkaloids, saponins, anthracene, glycosides, carbohydrates, proteins, amino acids, flavonoids, sterols, minerals, trace elements (iron, nickel, cobalt and iodine), vitamins, stigmast-en-3-beta, 27-diol-27 benzoate, glucotropoeolin, 4-methoxyglucobrassicin, sinapine, sinapic acid 
calmodulin, sinapoyglucose, esters of caffeic, p-coumaric, ferulic, quininc acids, 5-4'-dihydroxy-7,8,3', 5-tetramethoxyflavone, 5-3'-dihydroxy-7,8,4'trimethoxyflavone, 5-3'- dihydroxy-6,7,4'-trimethoxyflavone. Biological properties exhibit by this plant includes anti-inflammatory, anti-pyretic, analgesic, chemoprotective, anti-diabetic, anti-hypertensive, diuretic, hepatoprotective, wound healing, anti-diarrheal, anti-oxidant, hypercholesterolemic, nephroprotective, anti- bacterial, laxative, respiratory disorder healing, anti-arthritic, hypoglycaemic activity, anti-hyperlipidemic (Falana et al., 2014; Manohar et al., 2012; Ait-yahia et al., 2018; Shukla et al., 2015).

\section{Nigella sativa}

It is a member of family Ranunculaceae (Islam et al., 2017), also known with names such as Black cumin or kalaunji (Paarakh, 2010). Thymoquinone, thymohydroquinone, stigmastanol, dithymoquinone, p-cymene, carvacrol, 4- terpineol, t-anethol, sesquiterpene longifolene, $\alpha$-pinene and thymol are some of the important active compounds present in this plant (Ahmad et al., 2013) Some compounds are also present in trace quantity and includes two types of alkaloids- isoquinoline (nigellicimine and nigellicimine-N-oxide), and pyrazol alkaloids or indazole ring bearing alkaloids (nigellidine and nigellicine). Additional compounds include alpha-hederin, a water soluble pentacyclic triterpene and saponin (Al-Jassir, 1992). Pharmacological activities exhibited by this plant are anti-diabetic, anticancer, immunomodulator, analgesic, antimicrobial, anti-inflammatory, spasmolytic, bronchodilator, hepato-protective, renal protective, gastro-protective, anti-oxidant properties, anti-fungal, antischistosomiasis, Neuro-pharmacolgical activities, anti-asthmatic, anti-convulsant, anti-oxytocic, anti-fertility, anti-cancer, anti-nociceptive, anti-urolithatic, anxiolytic, anti-pyretic, cardiovascular, anti-allergic (Paarakh et al., 2010; Ahmad et al., 2013).

Table 1 Pharmacological aspects of different medicinal plants for cure of Chikungunya

Plant (Botanical Common Family Active constituents

Name)

Name

Family

Active constituents

Alkaloids, steroids,

Tinospor cordifolia

ae sesquiterpenoid, phenolics, aliphatic compounds, polysaccharides, diterpenoid lactones, and glycosides

\section{Zingiber officinale}

Ginger Zingiberaceae

Essential oils, phenolic compounds,

flavonoids, carbohydrates, proteins, steroids, terpenoids, and tannin alkaloids, lycosides, saponins,

Specified properties of plant for
Guduchi Menispermace treating Chikungunya

Immunomodulatory, Anti-pyretic,antiarthritic, anti-inflammatory, analgesic anti-allergic

Anti-inflammatory, anti-arthritic,Antipyretic, immunomoduatory, analgesic, anti-allergic
Diterpenoids, diterpene glycosides, Andrographis paniculata Kalmegh Acanthaceae lactones, flavanoids, and flavanoid glycosides, quinic acids, xanthones
Anti-viral, anti-inflammatory, antipyretic, analgesic anti-allergic, ,immunostimulatory

\section{References}

Ghosh and Saha, 2012;

Sharma et al., 2012;

Ashok et al., 2010;

Banerjee et al., 2018;

Pendse et al., 1997.

Dhanik et al., 2017;

Anosike et al., 2009;

Prakash et al., 2016

Mascolo et al., 1989

Puri et al., 2000.

Okhuarolo et al., 2014;

Jayakumar et al., 2013;

Wintachai et al., 2015;

Anbarasu et al., 2011;

Chandrasekaran et al., 2010; Puri et al., 1993.

Buddhadev et al., 2014;

Rahman et al., 2011;

Bano et al., 2017;

Mohan et al., 2011; linalool, urosolic acid, carvacro rosmarinic acid, apigenin,

Ocimum sanctum

Tulsi Lamiaceae

cirsimaritin, fatty acids, sitosterol, sugars (xylose and

polysaccharides), anthocyans
Immunomodulatory, anti-arthritic analgesic, Anti-pyretic, antiinflammatory
Vaghasiya et al., 2010;

Bawankule et al., 2015;

Ranjana and Tripathi, 2015; Kothari et al.
Hossain et al., 2014; 2012.

Anthocyanins, alkaloids, tannins, phenolics(chebulunic acid, ellagic acid and anthraquinones), polyphenols(corilagin, galloyl
Terminalia chebula

Harad Combretaceae glucose, punicalagin, terflavin A,

maslinic acid), fructose, amino acids, succinic acid, betasitosterol, resin, flavanol, glycosides, triterpenoids
Analgesic, anti-arthritic Antiinflammatory, larvicidal and ovicidal, immunomodulatory
Bag et al., 2013; Kaur et al., 2010; Nair et al., 2010; Yang et al., 2014; Veni et al., 2017; Aher and Wahi, 2011.
Emblica officinalis (Phyllanica emblica)
Polyphenols (gallic acid, ellagic acid, different tannins, minerals, vitamins, amino acids, fixed oil) flavonoids (rutin and quercetin), glycosides
Analgesic, anti-pyretic Antiinflammatory,immuno-modulatory, anti-diarrheal
Vimala et al., 2011;

Variya et al., 2016;

Gaire and Subedi, 2014;

Jain et al., 2015;

Perianayagam et al.,

2004; Golechha et al. Mehmood et al., 2011. 2014; Singh et al., 2013;

Kannan et al., 2009 (a);

Verma et al. ,2016; Devi
Anthraquinones and teir

Rubia cordifolia

Indian

Madder

Rubiaceae

glycosides, terpenes, bicyclic hexapeptides, iridoids, carboxylic acids and saccharides
Anti-arthritic, immunomodulatory, antiinflammation

Essential oils, flavanoids, terpenoids, mono-sesquiterpenes,

cyprotene, acopaene, cyperene,

Cyperus rotundus Nagarmot a
Cyperacea cyene, rotundene, valencen

rjunene trans-

calamenene, dcadinene
Anti-inflammatory, analgesic,antipyretic, mosquitoe repellent, antidiarrheal
Priya and Sirul, 2014;

Jaijesh et al., 2008;

Kannan et al., 2009(b);

Antarkar et al., 1983.

Imam et al., 2014

Peerzada et al., 2015;

Sundaram et al., 2008;

Gupta et al., 1971;

Singh et al., 2009; Uddin

et al., 2006. 
gmuurolene, cyperotundone, mustakone, isocyperol, acyperone,

4,11-selinnadien-3-one and 1,8cineole

Picrorhiza kurroa

Kutki
Scrophulariac

eae
Iridoids, acetophenones, cucurbitacins, picroside-I and II (major bioactive compounds), pikuroside, veronicoside, phenol glycosides, curcurbitacin 3methoxy-acetophenone glycosides and 4-hydroxy-
Anti-arthritic, immunomodulatory, analgesic, Anti-inflammatory
Masood et al., 2015; Sah

Kumar et al., 2013;

Salma et al., 2017;

Kumar et al., 2016 (a) ;

Gupta et al., 2006; Shid et al., 2013; Kumar et al., 2016 (b) and Varshney, 2015;

\begin{tabular}{|c|c|c|c|}
\hline Pluchea lanceolata & Rasana & Asteraceae & $\begin{array}{l}\text { Alkaloids, flavonoids, tannins, } \\
\text { steroids, glycosides, saponins, } \\
\text { terpenoids, proteins, carbohydrates, } \\
\text { phenols, lactones }\end{array}$ \\
\hline Terminalia bellerica & Baheda & Combretaceae & $\begin{array}{l}\text { Glucoside, tannins, gallic acid, } \\
\text { ethyl gallate, chebulinic acid, } \\
\text { lignans(termilignan and } \\
\text { thannilignan), 7-hydroxy } 3^{\prime} 4^{\prime} \\
\text { (methylenedioxy) flavone and } \\
\text { anolignanB, ellagic acid, galloyl } \\
\text { glucose, phyllemblin, beta- } \\
\text { sitosterol, mannitol, glucose, } \\
\text { fructose, rhamnose, alkaloids, } \\
\text { steroids, terpenoids, saponin }\end{array}$ \\
\hline Commiphora wightii & Guggul & Burseraceae & $\begin{array}{c}\text { Terpenoids (monoterpenoids, } \\
\text { sesquiterpenoids, triterpenoids), } \\
\text { steroids, flavonoids, guggultetrols, } \\
\text { lignans, sugars, amino acids. } \\
\text { Another photo constituents may } \\
\text { include long chain aliphatic tetrols, } \\
\text { aliphatic esters, ferulates, } \\
\text { carbohydrates, inorganic ions }\end{array}$ \\
\hline
\end{tabular}

Analgesic, anti-pyretic, immunological, immunomodulatory, anti-inflammatory

Anti-inflammatory,anti-arthritic

Arya and Patni, 2013

Srivastava and

Shanker, 2012; Chawla

et al., 1991; Chaturvedi and Singh, 1965.

Deb et al., 2016; Kadian et al., 2014; Kaur et al., 2010; Sharma et al., 2010; Saraphanchotiwitthaya et al., 2008.
Ani-viral, Anti-inflammatory, antiarthritic
Sarup et al., 2015;

Cunningham et al., 2018; Kunnumakkara $e t$ al., 2018; Francis et al., 2004; Gujral et al., 1960
Kocaadam and Çanlier, 2017; Khajehdehi et al. 2012; Li et al., 2011; Araújo and Leon, 2001; Joe et al., 2010; Çlkrikçl
Phenolic compounds, terpenoids, diarylheptanoids(curcuminoids),

Curcuma longa Turmeric Zingiberaceae diarylpentanoids, monoterpenes, sesquiterpwnws, diterpenes, triterpenes, alkaloid, and sterols
Anti-viral, immunomodulatory Antiinflammatory,analgesic et al., 2008; Perrone $e$ al., 2015; Mathew and Hsu, 2018; Gautam et al., 2007; Chainani- Wu, 2003; Ibironke and Owemidu, 2014.
Arora et al., 2013; Siddiqui, 2011; Harrasi et al., 2018; Menon and
Boswellia serrata

Indian frankince nse

Burseraceae acids
Monoterpenes, diterpenes, triterpenes, pentacyclic triterpenic ids (boswellic acids), tetracyclic triterpenic acids
Analgesic, anti-viral, anti-arthritic, antiinflammatory hein $e t$ al., 2006; Hamidpour et al., 2013; Bertocchi et al., 2018.

Viridiflorol, betacaryophyllene,sabinene, 4terpineol, gamma-terpinene,

Vitex negundo Nirgundi Verbenaceae caryophyllene oxide, 1-ocetan-3-ol, globulol[57], laonoid glycosides, negundoside, agnuside, vitegnoside, p-hydroxybenzoic acid, beta-sitosterol
Venkateshwarlu, 2012;

Singh et al., 2003;

Meena et al., 2011

Choudhary et al., 2015;

Anti-arthriic, analgesic, immunostimulatory anti-inflammatory, mosquito repellant

Ladda and Magdum,
2012; Gupta and

Tandon, 2005 . Hebbalkar et al., 1992.

Alkaloids, steroidal compounds, ergostane type typesteroidllactones, withaferin A, withanolides A-y, withasomniferin-A, withasomidienone,

Withania somnifera Ashwagan Solanaceae dha

withasomniferols A-C, withanone etc.Additional constituents include saponins, withanolides, withaniol,

acylsteryl glucosides, starc,

reducing sugar, hantreacetone, ducitol, amino acids

Gupta and Rana, 2007; Singh et al., 2007;

Anti-arthritic Immunomodulation, analgesic, anti-inflammatory
Singh et al., 2007;
Kumar et al., 2015

Jain et al., 2018; Ali et al., 2012; Bhavani,2015. 
glabrin A and B, glycyrrhetol, glabrolide, isoglabrolide,

isoflavones, coumarins, triterpene sterols, starches, pectins,

polysaccharides, simple sugars, gums, mucilage, amino acids, flavonoids, mineral salts, bitters, essential oil, fat, estroen, tannins, glycosides, protein, resins, volatile oils

Alkaloids, saponins, anthracene, glycosides, carbohydrates, proteins, amino acids, flavonoids, sterols, minerals, trace elements(iron,

nickel, cobalt and iodine), vitamins, stigmast-en-3-beta, 27-diol-27 benzoate, glucotropoeolin, 4Lepidium sativum Garden Brassicaceae methoxyglucobrassicin, sinapine, sinapic acid, calmodulin, sinapoyglucose, esters of caffeic, $\mathrm{p}$ coumaric, ferulic, quininc acids, 54'-dihydroxy-7,8,3', 5-

tetramethoxyflavone, 5-3'dihydroxy-7,8,4'-

trimethoxyflavone, 5-3'dihydroxy-6,7,4'-trimethoxyflavone Thymoquinone,

thymohydroquinone, stigmastanol,

dithymoquinone, p-cymene, carvacrol, 4-terpineol, t-anethol, sesquiterpene longifolene, $\alpha$-pinene and thymol

\begin{tabular}{|c|c|c|c|}
\hline Nigella sativa & $\begin{array}{l}\text { Black } \\
\text { cumin }\end{array}$ & $\begin{array}{c}\text { Ranunculacea } \\
\mathrm{e}\end{array}$ & $\begin{array}{l}\text { thymohydroquinone, stigmastanol, } \\
\text { dithymoquinone, p-cymene, } \\
\text { carvacrol, 4-terpineol, t-anethol, } \\
\text { sesquiterpene longifolene, } \alpha \text {-pinene }\end{array}$ \\
\hline
\end{tabular}

(immunomodulatory)

Damle 2014; Jitesh and Geetha,2017; Delbò, 2013.
Analgesic anti-diarrhea antiinflammatory
Falana et al., 2014

Manohar et al., 2012;

Ait-yahia et al., 2018;

Shukla et al., 2015; Al-

Yahya et al., 1994;

Manohar et al., 2009;

Raval et al., 2013.
Immunomodulatory, analgesic, antiallergic, anti-pyretic

\author{
Islam et al., 2017; \\ Paarakh, 2010; Ahmad \\ et al., 2013; Al-Jassir, \\ 1992 Paarakh et al., \\ 2010.
}

\section{CONCLUSION}

Chikungunya is a disease described as the 'to bend' condition of sufferers with joint pain and rash, caused by alpha virus. It's a viral disease affecting many individuals in India and abroad, but it's not a lethal infection and relevant or proper treatment can help to cure it. However, it's necessary to keep the adjacent areas of surroundings neat and clean so that the virus prevents from spreading. Several drugs for the treatment of chikungunya have been found and have recognized effect against chikungunya virus, although no specific antiviral treatment against infection is available. Treatment by herbal means provides the best way of preventing and curing chikungunya and helps sufferers to replenish or recover faster. Plants exhibit many biological and pharmacological properties which lighten the risks of chikungunya to a large extent and really help to cure it without any side effects reported.

Acknowledgement: We would like to acknowledge Dr. O.P. Bhalla Centra Library, Manav Rachna International Institute of Research and Studies for their valuable support.

\section{REFERENCES}

Aher, V., \& Wahi, A. (2011). Immunomodulatory Activity of Alcohol Extract of Terminalia chebula Retz Combretaceae. Tropical Journal of Pharmaceutical Research, 10(5). https://doi.org/ 10.4314/tipr.v10i5.5

Ahmad, A., Husain, A., Mujeeb, M., Khan, S. A., Najmi, A. K., Siddique, N. A., ... Anwar, F. (2013). A review on therapeutic potential of Nigella sativa: A miracle herb. Asian Pacific Journal of Tropical Biomedicine, 3(5), 337-352. https://doi.org/ 10.1016/s2221-1691(13)60075-1

Ait-yahia Ouahiba, Perreau, F., Bouzroura, S.-A., Benmalek, Y., Dob, T., \& Belkebir, A. (2018). Chemical composition and biological activities of n-butano extract of Lepidium sativum L (Brassicaceae) seed. Tropical Journal of Pharmaceutical Research, 17(5), 891. https://doi.org/ 10.4314/tjpr.v17i5.20

Al-Harrasi, A., Rehman, N. U., Khan, A. L., Al-Broumi, M., Al-Amri, I., Hussain, J., ... Csuk, R. (2018). Chemical, molecular and structural studies of Boswellia species: $\beta$-Boswellic Aldehyde and 3-epi-11 $\beta$-Dihydroxy BA as precursors in biosynthesis of boswellic acids. PLOS ONE, 13(6), e0198666. https://doi.org/ 10.1371/journal.pone.0198666

Ali Siddiq, N., Singh, S., Mairaj Sid, M., \& Husain Kha, T. (2012). Immunomodulatory Effect of Withania somnifera, Asparagus racemosus and Picrorhiza kurroa Roots. International Journal of Pharmacology, 8(2), 108-114 https://doi.org/10.3923/ijp.2012.108.114

Al-Jassir, M. S. (1992). Chemical composition and microflora of black cumin (Nigella sativa L.) seeds growing in Saudi Arabia. Food Chemistry, 45(4), 239 242. https://doi.org/ 10.1016/0308-8146(92)90153-s
Al-Yahya, M. A., Mossa, J. S., Ageel, A. M., \& Rafatullah, S. (1994) Pharmacological and safety evaluation studies on Lepidium sativum L., Seeds. Phytomedicine, 1(2), 155-159. https://doi.org/ 10.1016/s0944-7113(11)80035-8

Amin, M.R., Rahman, M.M., Islam, Q.T. 2017. Chikungunya. Journal of Medicine, 18(2), 92-108. https://doi.org/ 10.3329/jom.v18i2.33687

Anbarasu, K., Manisenthil, K. K., \& Ramachandran, S. (2011). Antipyretic, antiinflammatory and analgesic properties of nilavembu kudineer choornam: a classical preparation used in the treatment of chikungunya fever. Asian Pacific Journal of Tropical Medicine, 4(10), 819-823. https://doi.org/ 10.1016/s19957645(11)60201-0

Anosike, C. ., \& Obidoa, O. (2010). Anti-inflammatory and anti-ulcerogenic effect of ethanol extract of coconut (Cocos nucifera) on experimental rats. African Journal of Food, Agriculture, Nutrition and Development, 10(10). https://doi.org/ 10.4314/ajfand.v10i10.62910

Antarkar, S. S., Chinwalla, T., \& Bhatt, N. (1983). Anti-inflammatory activity of Rubia cordifolia Linn. in rats. Indian J. Pharmacol, 15, 185-188.

Araújo, C., \& Leon, L. (2001). Biological activities of Curcuma longa L. Memórias Do Instituto Oswaldo Cruz, 96(5), 723-728. https://doi.org/ 10.1590/s0074-02762001000500026

Arora, R., Malhotra, P., Sharma, A., Haniadka, R., Yashawanth, H.S., \& Baliga, M.S. (2013). Medicinal efficacy of Indian herbal remedies for the treatment of arthritis. In: Bioactive Food as Dietary Interventions for Arthritis and Related Inflammatory Diseases, Academic Press, pp. 601-617

Arya, D., \& Patni, V. (2013). Pharmacognostic profile and phytochemical investigation of Pluchea lanceolata Oliver \& Hiern. in vivo and in vitro. Int. J. Pharm. Sci. Rev. Res, 22(2), 157-161.

Ashok, B., Ravishankar, B., Prajapati, P., \& Bhat, S. (2010). Antipyretic activity of Guduchi Ghrita formulations in albino rats. AYU (An International Quarterly Journal of Research in Ayurveda), 31(3), 367. https://doi.org/ 10.4103/0974$\underline{8520.77162}$

Bag, A., Bhattacharyya, S. K., \& Chattopadhyay, R. R. (2013). The development of Terminalia chebula Retz. (Combretaceae) in clinical research. Asian Pacific Journal of Tropical Biomedicine, 3(3), 244-252. https://doi.org/ 10.1016/s22211691(13)60059-3

Banerjee, N., Saha, B., \& Mukhopadhyay, S. (2018). Intracellular ROS generated in chikungunya patients with persisting polyarthralgia can be reduced by Tinospora cordifolia leaf extract. VirusDisease, 29(3), 375-379. https://doi.org/ 10.1007/s13337-018-0465-1

Bano, N., Ahmed, A., Tanveer, M., Khan, G. M., \& Ansari, M. T. (2017). Pharmacological evaluation of Ocimum sanctum. Journal of Bioequivalence and Bioavailability, 9(3), 387-492. https://doi.org/10.4172/jbb.1000330

Bawankule, D., Kumar, A., Agarwal, K., Maurya, A., Shanker, K., Bushra, U., \& Tandon, S. (2015). Pharmacological and phytochemical evaluation of Ocimum sanctum root extracts for its antiinflammatory, analgesic and antipyretic activities. Pharmacognosy Magazine, 11(42), 217. https://doi.org/10.4103/0973$\underline{1296.157743}$ 
Bernard, E., Solignat, M., Gay, B., Chazal, N., Higgs, S., Devaux, C., \& Briant, L. (2010). Endocytosis of Chikungunya Virus into Mammalian Cells: Role of Clathrin and Early Endosomal Compartments. PLoS ONE, 5(7), e11479. https://doi.org/ 10.1371/journal.pone.0011479

Bertocchi, M., Isani, G., Medici, F., Andreani, G., Tubon Usca, I., Roncada, P., Bernardini, C. (2018). Anti-Inflammatory Activity of Boswellia serrata Extracts: An In Vitro Study on Porcine Aortic Endothelial Cells. Oxidative Medicine and Cellular Longevity, 2018, 1-9. https://doi.org/ $10.1155 / 2018 / 2504305$

Bhavani, S. (2015). Review on Anti-Pyretics \& Analgesic Herbs in Siddha Medicine. Journal of Pharmaceutical Sciences and Research, 7(10) ,812-817.

Buddhadev, S. G., Buddhadev, S. S., \& Mehta, N. D. (2014). A review article on Ocimum sanctum Linn. Int. Peer Revd. Ayur. J, 2(2), 1-6.

Cavrini, F., Gaibani, P., Pierro, A. M., Rossini, G., Landini, M. P., \& Sambri, V. (2009). Chikungunya: an emerging and spreading arthropod-borne viral disease. The Journal of Infection in Developing Countries, 3(10), 744-752. https://doi.org/ 10.3855/jidc.169

Cikrikci, S., Mozioglu, E., \& Yilmaz, H. (2008). Biological activity of curcuminoids isolated from Curcuma longa. Records of Natural Products, 2(1), $19-24$

Cunha, R. V. da, \& Trinta, K. S. (2017). Chikungunya virus: clinical aspects and treatment - A Review. Memórias Do Instituto Oswaldo Cruz, 112(8), 523-531. https://doi.org/ 10.1590/0074-02760170044

Cunningham, A. B., Brinckmann, J. A., Kulloli, R. N., \& Schippmann, U. (2018) Rising trade, declining stocks: the global gugul (Commiphora wightii) trade. Journal of ethnopharmacology, 22-32. https://doi.org/10.1016/j.jep.2018.04.040

Damle, M. (2014). Glycyrrhiza glabra (Liquorice)-a potent medicinal herb. International journal of herbal medicine, 2(2), 132-136.

Deb, A., Barua, S., \& Das, B. (2016). Pharmacological activities of Baheda (Terminalia bellerica): a review. Journal of pharmacognosy and phytochemistry, 5(1), 194-197.

Delbò, R.M. (2013). Assessment report on Glycyrrhiza glabra L. and/or Glycyrrhiza inflata Bat. and/or Glycyrrhiza uralensis Fisch., radix. European Medicines Agency, EMA/HMPC/571122/2010 Corr. Accessed 12 March 2013.

Devi Priya, M., \& Siril, E. A. (2014). Traditional and modern use of indian madder (Rubia cordifolia L.): an overview. Int. J. Pharm. Sci. Rev. Res, 25(1), 154-164.

Dhanik, J., Arya, N., \& Nand, V. (2017). A Review on Zingiber officinale. Journal of Pharmacognosy and Phytochemistry, 6(3), 174-184.

Falana, H., Nofal, W., \& Nakhleh, H. (2014). A Review Article Lepidium Sativum (Garden cress).

Francis, J., Raja, S., \& Nair, M. (2004). Bioactive Terpenoids and Guggulusteroids fromCommiphora mukul Gum Resin of Potential AntiInflammatory Interest. Chemistry \& Biodiversity, 1(11), 1842-1853. https://doi.org/10.1002/cbdv.200490138

Gaire, B. P., \& Subedi, L. (2014). Phytochemistry, pharmacology and medicina properties of Phyllanthus emblica Linn. Chinese Journal of Integrative Medicine. https://doi.org/10.1007/s11655-014-1984-2

Galán-Huerta, K. A., Rivas-Estilla, A. M., Fernández-Salas, I., Farfan-Ale, J. A., \& Ramos-Jiménez, J. (2015). Chikungunya virus: A general overview. Medicina Universitaria, 17(68), 175-183. https://doi.org/10.1016/j.rmu.2015.06.001

Ganesan, V., Duan, B., \& Reid, S. (2017). Chikungunya Virus: Pathophysiology, Mechanism, and Modeling. Viruses, 9(12), 368 https://doi.org/10.3390/v9120368

Gautam, S. C., Gao, X., \& Dulchavsky, S. (n.d.). Immunomodulation by Curcumin. The Molecular Targets and Therapeutic Uses of Curcumin in Health and Disease, 321-341. https://doi.org/10.1007/978-0-387-46401-5_14

Ghosh, S., \& Saha, S. (2012). Tinospora cordifolia: One plant, many roles Ancient Science of Life, 31(4), 151. https://doi.org/10.4103/0257-7941.107344

Golechha, M., Sarangal, V., Ojha, S., Bhatia, J., \& Arya, D. S. (2014). AntiInflammatory Effect of Emblica officinalis in Rodent Models of Acute and Chronic Inflammation: Involvement of Possible Mechanisms. International Journal of Inflammation, 2014, 1-6. https://doi.org/10.1155/2014/178408 Gujral, M. L., Sareen, K., Tangri, K. K., Amma, M. K., \& Roy, A. K. (1960) Antiarthritic and anti-inflammatory activity of gum guggul (Balsamodendron mukul Hook). Indian journal of physiology and pharmacology, 4, 267-273.

Gupta, A., Khajuria, A., Singh, J., Bedi, K. L., Satti, N. K., Dutt, P., ... Qazi, G. N. (2006). Immunomodulatory activity of biopolymeric fraction RLJ-NE-205 from Picrorhiza kurroa. International Immunopharmacology, 6(10), 1543-1549. https://doi.org/10.1016/j.intimp.2006.05.002

Gupta, G. L., \& Rana, A. C. (2007). Withania somnifera (Ashwagandha): A review. Pharmacognosy Reviews, 1(1), 129-136

Gupta, M. B., Palit, T. K., Singh, N., \& Bhargava, K. P. (1971). Pharmacological studies to isolate the active constituents from Cyperus rotundus possessing antiinflammatory, anti-pyretic and analgesic activities. The Indian journal of medical research, 59(1), 76

Gupta, R. K., \& Tandon, V. R. (2005). Antinociceptive activity of Vitex-negundo Linn leaf extract. Indian journal of physiology and pharmacology, 49(2), 163170.
Hamidpour, R., Hamidpour, S., Hamidpour, M., \& Shahlari, M. (2013). Frankincense (乳香 Rǔ Xiāng; Boswellia Species): From the Selection of Traditional Applications to the Novel Phytotherapy for the Prevention and Treatment of Serious Diseases. Journal of Traditional and Complementary Medicine, 3(4), 221-226. https://doi.org/10.4103/2225-4110.119723

Hebbalkar, D. S., Hebbalkar, G. D., Sharma, R. N., Joshi, V. S., \& Bhat, V. S. (1992). Mosquito repellent activity of oils from Vitex negundo Linn. leaves. The Indian Journal of Medical Research, 95, 200-203.

Hossain, M. S., Urbi, Z., Sule, A., \& Rahman, K. M. H. (2014). Andrographis paniculata(Burm. f.) Wall. ex Nees: A Review of Ethnobotany, Phytochemistry, and Pharmacology. The Scientific World Journal, 2014, 1-28 doi:10.1155/2014/274905

https://doi.org/10.3923/ajdd.2011

Chainani-Wu, N. (2003). Safety and Anti-Inflammatory Activity of Curcumin: A Component of Tumeric (Curcuma longa). The Journal of Alternative and Complementary Medicine, 9(1), 161-168. https://doi.org/ $10.1089 / 107555303321223035$

Chandrasekaran, C. V., Gupta, A., \& Agarwal, A. (2010). Effect of an extract of Andrographis paniculata leaves on inflammatory and allergic mediators in vitro. Journal of ethnopharmacology, 129(2), 203-207.

Chaturvedi, G. N., \& Singh, R. H. (1965). EXPERIMENTAL STUDIES ON THE ANTIARTHRITIC EFFECT OF CERTAIN INDIGENOUS DRUGS. The Indian Journal of Medical Research, 53, 71-80.

Chawla, A. S., Kaith, B. S., Handa, S. S., Kulshreshtha, D. K., \& Srimal, R. C. (1991). Chemical investigation and anti-inflammatory activity of Pluchea lanceolata. Fitoterapia, 62(5), 441-444.

Chhabra, M., Mittal, V., Bhattacharya, D., Rana, U., \& Lal, S. (2008) Chikungunya fever: A re-emerging viral infection. Indian Journal of Medical Microbiology, 26(1), 5. https://doi.org/ 10.4103/0255-0857.38850

Choudhary, M., Kumar, V., Malhotra, H., \& Singh, S. (2015). Medicinal plants with potential anti-arthritic activity: Journal of Intercultural Ethnopharmacology, 4(2), 147. https://doi.org/ 10.5455/jice.20150313021918

Ibironke, G. F., \& Owemidu, I. O. (2014). Analgesic activity of the ethanol extract of Curcuma longa rhizome and its mechanism of action. Nigerian journal of physiological sciences: official publication of the Physiological Society of Nigeria, 29(1), 67-70.

Imam, H., Lone, A., Seikh, A., Sofi, G., \& Zarnigar. (2014). The incredible benefits of Nagarmotha (Cyperus rotundus). International Journal of Nutrition, Pharmacology, Neurological Diseases, 4(1), 23. https://doi.org/10.4103/22310738.124611

Islam, M.T., Guha, B., Hosen, S., Riaz, T.A., Shahadat, S. (2017). Nigellalogy: A Review on Nigella sativa. MOJ Bioequivalence \& Bioavailability, 3(6), 167-181. https://doi.org/10.15406/mojbb.2017.03.00056

Jaijesh, P., Srinivasan, K. K., Bhagath Kumar, P., Sreejith, G., \& Ciraj, A. M (2008). Anti arthritic property of the plant Rubia cordifolia lin. Pharmacologyonline, 1, 107-113.

Jain, J., Narayanan, V., Chaturvedi, S., Pai, S., Sunil, S. 2018. In Vivo Evaluation of Withania somnifera-Based Indian Traditional Formulation (Amukkara Choornam), Against Chikungunya Virus-Induced Morbidity and Arthralgia Journal of evidence-based integrative medicine, 23, 1-7. https://doi.org/10.1177/2156587218757661

Jain, R., Pandey, R., Mahant, R. N., \& Rathore, D. S. (2015). A review on medicinal importance of Emblica officinalis. International Journal of Pharmaceutical Sciences and Research, 6(1), 72

Jayakumar, T., Hsieh, C.-Y., Lee, J.-J., \& Sheu, J.-R. (2013). Experimental and Clinical Pharmacology ofAndrographis paniculataand Its Major Bioactive Phytoconstituent Andrographolide. Evidence-Based Complementary and Alternative Medicine, 2013, 1-16. https://doi.org/10.1155/2013/846740

Jitesh, S., \& Geetha, R. V. (2017). Anti Inflammatory Activity of Glycrrhiza glabra Extract-An in vitro Study. Journal of Pharmaceutical Sciences and Research, 9(4), 451-452.

Joe, B., Vijaykumar, M., \& Lokesh, B. R. (2004). Biological properties of curcumin-cellular and molecular mechanisms of action. Critical reviews in food science and nutrition, 44(2), 97-111. https://doi.org/10.1080/10408690490424702

Kadian, R., Parle, M., \& Yadav, M. (2014). Therapeutic potential and phytopharmacology of terminalia bellerica. World journal of pharmacy and pharmaceutical sciences, 3(10), 804-806.

Kannan, M., Singh, A. J. A., \& Narayanan, M. (2009). Phytochemistry and ethanopharmacological studies on Rubia cordifolia Linn.(Rubiaceae). Ethnobotanical Leaflets, 2009(2), 9.

Kannan, M., Singh, R., \& Narayanan, M. (2009). Phytochemistry and immunopharmacological investigation of Rubia cordifolia Linn.(Rubiaceae) Pharmacology, 3, 653-62

Kaur, S., \& Jaggi, R. K. (2010). Antinociceptive activity of chronic administration of different extracts of Terminalia bellerica Roxb. and Terminalia chebula Retz. fruits.. Indian Journal of Experimental Biology, 48(9), 925-930.

Khajehdehi, P. (2012). Turmeric: Reemerging of a Neglected Asian Traditional Remedy. Journal of Nephropathology, 1(1), 17-22. https://doi.org/10.5812/jnp.5 
Kocaadam, B., \& Şanlier, N. (2015). Curcumin, an active component of turmeric (Curcuma longa), and its effects on health. Critical Reviews in Food Science and Nutrition, 57(13), 2889-2895. https://doi.org/10.1080/10408398.2015.1077195 Kothari, A., \& Sharma, S. (2012). Evaluation of anti-inflammatory effect of fresh tulsi leaves (Ocimum Sanctum) against different mediators of inflammation in albino rats. Int J Pharm Sci Rev Res, 14, 119-23.

Kumar, N., Kumar, T., \& Sharma, S. K. (2013). Phytopharmacological review on genus picrorhiza. Int J Universal Pharm Bio Sci, 2(4), 334-347.

Kumar, R.(a), Gupta, Y. K., Singh, S., \& Arunraja, S. (2016). Picrorhiza kurroa Inhibits Experimental Arthritis Through Inhibition of Pro-inflammatory Cytokines, Angiogenesis and MMPs. Phytotherapy Research, 30(1), 112-119. https://doi.org/10.1002/ptr.5509

Kumar, R.(b), Gupta, Y., Singh, S., \& Raj, A. (2016). Anti-inflammatory Effect of Picrorhiza kurroa in Experimental Models of Inflammation. Planta Medica 82(16), 1403-1409. https://doi.org/10.1055/s-0042-106304

Kumar, V., Dey, A., Hadimani, M. B., Marcovic, T., \& Emerald, M. (2015) Chemistry and pharmacology of withania somnifera: An update. Tang [Humanitas Medicine], 5(1), 1.1-1.13. https://doi.org/10.5667/tang.2014.0030 Kunnumakkara, A. B., Banik, K., Bordoloi, D., Harsha, C., Sailo, B. L. Padmavathi, G., ... Aggarwal, B. B. (2018). Googling the Guggul (Commiphora and Boswellia) for Prevention of Chronic Diseases. Frontiers in Pharmacology, 9. https://doi.org/10.3389/fphar.2018.00686

Ladda, P. L., \& Magdum, C. S. (2012). Vitex negundo Linn.: Ethnobotany, phytochemistry and pharmacology-A review. International Journal of Advances in Pharmacy, Biology and Chemistry, 1(1), 111-120.

Li, S. (2011). Chemical Composition and Product Quality Control of Turmeric (Curcuma longa L.). Pharmaceutical Crops, 5(1), 28-54 https://doi.org/10.2174/2210290601102010028

Manohar, D., Lakshman, K., Shylaja, H., Viswanatha, G. L., Rajesh, S., \& Nandakumar, K. (2009). Antidiarrheal activity of methanolic extracts of seeds of Lepidium sativum. Journal of Natural Remedies, 9(2), 197 201.https://doi.org/10.18311/jnr/2009/240

Manohar, D., Viswanatha, G. L., Nagesh, S., Jain, V., \& Shivaprasad, H. N. (2012). Ethnopharmacology of Lepidium sativum Linn (Brassicaceae): a review. International journal of phytothearpy research, 2(1), 1-7.

Mascolo, N., Jain, R., Jain, S. C., \& Capasso, F. (1989). Ethnopharmacologic investigation of ginger (Zingiber officinale). Journal of Ethnopharmacology, 27(1-2), 129-140. https://doi.org/10.1016/0378-8741(89)90085-8

Masood, M. (2015). Picrorhiza kurroa: An ethnopharmacologically importan plant species of Himalayan region. Pure and Applied Biology, 4(3), 407-417. https://doi.org/10.19045/bspab.2015.43017

Mathew, D., \& Hsu, W.-L. (2018). Antiviral potential of curcumin. Journal of Functional Foods, 40, 692-699. https://doi.org/10.1016/j.jff.2017.12.017

Meena, A. K., Niranjan, U. S., Rao, M. M., Padhi, M. M., \& Babu, R. (2011). A review of the important chemical constituents and medicinal uses of Vitex genus. Asian Journal of Traditional Medicines, 6(2), 54-60.

Mehmood, M. H., Siddiqi, H. S., \& Gilani, A. H. (2011). The antidiarrheal and spasmolytic activities of Phyllanthus emblica are mediated through dual blockade of muscarinic receptors and $\mathrm{Ca} 2+$ channels. Journal of Ethnopharmacology, 133(2), 856-865. https://doi.org/10.1016/j.jep.2010.11.023

Menon, M., \& Kar, A. (1971). Analgesic and Psychopharmacological effects of the gum resin of Boswellia serrata. Planta Medica, 19(02), 333-341. https://doi.org/10.1055/s-0028-1099651

Mohan, A., Kiran, D. ., Manohar, C., \& Kumar, P. (2010). Epidemiology, clinica manifestations, and diagnosis of chikungunya fever: Lessons learned from the reemerging epidemic. Indian Journal of Dermatology, 55(1), 54 https://doi.org/10.4103/0019-5154.60355

Mohan, L., Amberkar, M. V., \& Kumari, M. (2011). Ocimum sanctum Linn (Tulsi) - an overview. Int J Pharm Sci Rev Res, 7(1), 51-53.

Nair, V., Singh, S., \& Gupta, Y. K. (2010). Anti-arthritic and disease modifying activity ofTerminalia chebulaRetz. in experimental models. Journal of Pharmacy and Pharmacology, 62(12), 1801-1806. https://doi.org/10.1111/j.20427158.2010.01193.x

Nesar, A., Khalid, M., Juber, A., Mujahid, M., Badruddin, M. A., \& Nazma, K (2016). Glycyrrhiza glabra: for traditional uses and pharmacological actions. Advanced Journal of Pharmacie and Life Science Research, 4(2), 23-32.

Okhuarobo, A., Ehizogie Falodun, J., Erharuyi, O., Imieje, V., Falodun, A., \& Langer, P. (2014). Harnessing the medicinal properties of Andrographis paniculata for diseases and beyond: a review of its phytochemistry and pharmacology. Asian Pacific Journal of Tropical Disease, 4(3), 213-222. https://doi.org/10.1016/s2222-1808(14)60509-0

Paarakh, P. M. (2010). Nigella sativa Linn.-A comprehensive review. Indian Journal of Natural Products and Resources, 1(4), 409-429.

Peerzada, A. M., Ali, H. H., Naeem, M., Latif, M., Bukhari, A. H., \& Tanveer, A (2015). Cyperus rotundus L.: Traditional uses, phytochemistry, and pharmacological activities. Journal of Ethnopharmacology, 174, 540-560. https://doi.org/10.1016/j.jep.2015.08.012

Pendse, V. K., Dadhich, A. P., Mathur, P. N., Bal, M. S., \& Madan, B. R. (1977) Antiinflammatory, immunosuppressive and some related pharmacological actions of the water extract of Neem Giloe (Tinospora cordifolia): A preliminary report Indian journal of pharmacology, 9(3), 221.

Perianayagam, J. B., Sharma, S. K., Joseph, A., \& Christina, A. J. M. (2004) Evaluation of anti-pyretic and analgesic activity of Emblica officinalis Gaertn Journal of Ethnopharmacology, 95(1), 83-85. doi:10.1016/j.jep.2004.06.020

Perrone, D., Ardito, F., Giannatempo, G., Dioguardi, M., Troiano, G., Lo Russo, L., ... \& Lo Muzio, L. (2015). Biological and therapeutic activities, and anticancer properties of curcumin. Experimental and therapeutic medicine, 10(5), 1615-1623. https://doi.org/10.3892/etm.2015.2749

Prakash, D., Katiyar, N.S., Singh, A.P., Gangwar, A.K. (2016). Evaluation of anti- arthritic potential of Zingiber officinale in Experimental rats. European journal of pharmaceutical and medical research, 3(4), 305-308.

Puri, A., Sahai, R., Singh, K. L., Saxena, R. ., Tandon, J. ., \& Saxena, K. . (2000) Immunostimulant activity of dry fruits and plant materials used in Indian traditional medical system for mothers after child birth and invalids. Journal of Ethnopharmacology, 71(1-2), 89-92. https://doi.org/10.1016/s03788741(99)00181-6

Puri, A., Saxena, R., Saxena, R. P., Saxena, K. C., Srivastava, V., \& Tandon, J. S. (1993). Immunostimulant Agents from Andrographis paniculata. Journal of Natural Products, 56(7), 995-999. https://doi.org/10.1021/np50097a002

Rahman, S., Islam, R., Kamruzzaman, M., Alam, K., \& Jamal, A. H. M. (2011) Ocimum sanctum L.: A review of phytochemical and pharmacological profile. American Journal of Drug Discovery and Development, 1-5.

Rahman, S., Suchana, S. A., Rashid, S. S., \& Pavel, O. F. (2017). A review article on Chikungunya virus. World Journal of Pharmaceutical Research, 6(13), 100-107. https://doi.org/ 10.20959/wjpr201713-9851

Ramachandran, V., Kaur, P., Kanagasabai, K., Vadivoo, S., \& Murhekar, M (2014). Persistent arthralgia among Chikungunya patients and associated risk factors in Chennai, South India. Journal of Postgraduate Medicine, 60(1), 3 https://doi.org/10.4103/0022-3859.128795

Ranjana, T., \& Tripathi, V. D. (2015). Therapeutic effect of Tulsi (Ocimum sanctum Linn) in general and oral health _. Ayurlog: National Journal of Research in Ayurve d Science, 3, 1-12.

Raval, N., Ravishankar, B., \& Ashok, B. (2013). Anti-inflammatory effect of Chandrashura (Lepidium sativum Linn.) an experimental study. $A Y U$ ( $A n$ International Quarterly Journal of Research in Ayurveda), 34(3), 302 https://doi.org/10.4103/0974-8520.123132

Sah, J. N., \& Varshney, V. K. (2013). Chemical constituents of Picrorhiza genus. American Journal of Essential Oils and Natural Products, 1(2), 22-37.

Salma, U., Kundu, S., Gantait, S. 2017. Phytochemistry and Pharmaceutical Significance of Picrorhiza kurroa Royle ex Benth. In Phytochemistry and Pharmacology of Medicinal Herbs. pp.26-37.

Saraphanchotiwitthaya, A., Sripalakit, P., Ingkaninan, K. (2008). Effects of Terminalia bellerica Roxb. methanolic extract on mouse immune response in vitro. Maejo international journal of science and technology, 2(02), 400-407.

Sarup, P., Bala, S., \& Kamboj, S. (2015). Pharmacology and Phytochemistry of Oleo-Gum Resin of Commiphora wightii (Guggulu). Scientifica, 2015, 1-14. https://doi.org/:10.1155/2015/138039

Sharma, S.K., Jain, S. (2018). Chikungunya: a rheumatologist's perspective. International journal of rheumatic diseases, 21(3), 584-601. https://doi.org/10.1111/1756-185X.13273

Sharma, U. S., Sharma, U. K., \& Singh, A. (2010). Screening of Terminalia bellirica fruits extracts for its analgesic and antipyretic activities. Jordan Journal of Biological Sciences, 147(615), 1-4.

Sharma, U., Bala, M., Kumar, N., Singh, B., Munshi, R. K., \& Bhalerao, S. (2012). Immunomodulatory active compounds from Tinospora cordifolia Journal of Ethnopharmacology, 141(3), 918-926. https://doi.org/10.1016/j.jep.2012.03.027

Shid, R. L., Raha, S. B., \& Shid, S. L. (2013). Evaluation of analgesic activity of roots of Picrorhiza kurroa. Journal of Drug Delivery and Therapeutics, 3(4). https://doi.org/10.22270/jddt.v3i4.557

Shukla. A.K., Bigoniya, P., Soni, P. (2015). Hypolipidemic activity of Lepidium sativum Linn. seed in rats. IOSR Journal of Pharmacy and Biological Sciences, 10(4), 13-22. https://doi.org/10.6084/m9.figshare.1518514.v1

Schilte, C., Staikovsky, F., Couderc, T., Madec, Y., Carpentier, F., Kassab, S., ... Michault, A. (2013). Chikungunya Virus-associated Long-term Arthralgia: A 36 month Prospective Longitudinal Study. PLoS Neglected Tropical Diseases, 7(3), e2137. https://doi.org/10.1371/journal.pntd.0002137

Schwartz, O., \& Albert, M. L. (2010). Biology and pathogenesis of chikungunya virus. Nature Reviews Microbiology, 8(7), 491-500 https://doi.org/10.1038/nrmicro2368

Siddiqui, M. Z. (2011). Boswellia serrata, a potential antiinflammatory agent: an overview. Indian journal of pharmaceutical sciences, 73(3), 255 https://doi.org/10.4103/0250-474X.93507

Singh, G., Sharma, P. K., Dudhe, R., \& Singh, S. (2010). Biological activities of Withania somnifera. Ann Biol Res, 1(3), 56-63.

Singh, M. K., Yadav, S. S., Gupta, V., \& Khattri, S. (2013). Immunomodulatory role of Emblica officinalis in arsenic induced oxidative damage and apoptosis in thymocytes of mice. BMC Complementary and Alternative Medicine, 13(1) https://doi.org/10.1186/1472-6882-13-193 
Singh, S. K., \& Unni, S. K. (2011). Chikungunya virus: host pathogen interaction. Reviews in Medical Virology, 21(2), 78-88. https://doi.org/10.1002/rmv.681

Singh, S. P., Raghavendra, K., \& Dash, A. P. (2009). Evaluation of Hexane Extract of Tuber of Root of Cyperus rotundus Linn (Cyperaceae) for Repellency against Mosquito Vectors. Journal of Parasitology Research, 2009, 1-5. https://doi.org/10.1155/2009/908085

Singh, V., Dayal, R., \& Bartley, J. P. (2003). Chemical constituents of Vitex negundo leaves. J Med Arom Plant Sci, 25(1), 94-98.

Srivastava, P., \& Shanker, K. (2012). Pluchea lanceolata (Rasana): Chemical and biological potential of Rasayana herb used in traditional system of medicine. Fitoterapia, 83(8), 1371-1385. https://doi.org/10.1016/j.fitote.2012.07.008

Staples, J. E., Breiman, R. F., \& Powers, A. M. (2009). Chikungunya Fever: An Epidemiological Review of a Re-Emerging Infectious Disease. Clinical Infectious Diseases, 49(6), 942-948. https://doi.org/10.1086/605496

Strauss, J. H., \& Strauss, E. G. (1994). The alphaviruses: gene expression, replication, and evolution. Microbiol. Mol. Biol. Rev., 58(3), 491-562.

Sundaram, M. S., Sivakumar, T., \& Balamurugan, G. (2008). Anti-inflammatory effect of Cyperus rotundus Linn. Leaves on acute and subacute inflammation in experimental rat models. Biomedicine, 28, 302-304.

Teo, T.-H., Lum, F.-M., Claser, C., Lulla, V., Lulla, A., Merits, A., ... Ng, L. F. P. (2012). A Pathogenic Role for CD4+ T Cells during Chikungunya Virus Infection in Mice. The Journal of Immunology, 190(1), 259-269. https://doi.org/:10.4049/jimmunol.1202177

Thiberville, S.-D., Moyen, N., Dupuis-Maguiraga, L., Nougairede, A., Gould, E. A., Roques, P., \& de Lamballerie, X. (2013). Chikungunya fever: Epidemiology, clinical syndrome, pathogenesis and therapy. Antiviral Research, 99(3), 345-370. https://doi.org/10.1016/j.antiviral.2013.06.009

Uddin, S. J., Mondal, K., Shilpi, J. A., \& Rahman, M. T. (2006). Antidiarrhoeal activity of Cyperus rotundus. Fitoterapia, 77(2), 134-136. https://doi.org/10.1016/j.fitote.2004.11.011

Vaghasiya, J., Datani, M., Nandkumar, K., Malaviya, S., \& Jivani, N. (2010) Comparative evaluation of alcoholic and aqueous extracts of Ocimum sanctum for immunomodulatory activity. Int J Pharm Biol Res, 1(1), 25e9.

Variya, B. C., Bakrania, A. K., \& Patel, S. S. (2016). Emblica officinalis (Amla) A review for its phytochemistry, ethnomedicinal uses and medicinal potentials with respect to molecular mechanisms. Pharmacological Research, 111, 180-200. https://doi.org/10.1016/j.phrs.2016.06.013

Veni, T., Pushpanathan, T., \& Mohanraj, J. (2016). Larvicidal and ovicidal activity of Terminalia chebula Retz. (Family: Combretaceae) medicinal plant extracts against Anopheles stephensi, Aedes aegypti and Culex quinquefasciatus. Journal of Parasitic Diseases, 41(3), 693-702. https://doi.org/10.1007/s12639016-0869-z

Venkateswarlu, K. (2012). Vitex negundo: Medicinal values, Biological activities, Toxicity studies and Phytopharmacological actions. Int J Pharm Phytopharmacol Res, 2(2), 126-133.

Verma, A., Kumar, B., Alam, P., Singh, V., \& Gupta, S. K. (2016). Rubia cordifolia-a review on pharmaconosy and phytochemistry. International Journal of Pharmaceutical Sciences and Research, 7(7), 2720-2731. https://doi.org/10.13040/IJPSR.0975-8232.

Vimala, Y., Rachel, K. V., Pramodini, Y., \& Umasankar, A. (2011). Usage of Indian Gooseberry (Emblica officinalis) Seeds in Health and Disease. Nuts and Seeds in Health and Disease Prevention, 663-670. https://doi.org/10.1016/b9780-12-375688-6.10077-5

Von Rhein, C., Weidner, T., Henß, L., Martin, J., Weber, C., Sliva, K., \& Schnierle, B. S. (2016). Curcumin and Boswellia serrata gum resin extract inhibit chikungunya and vesicular stomatitis virus infections in vitro. Antiviral Research, 125, 51-57. https://doi.org/10.1016/j.antiviral.2015.11.007

Wintachai, P., Kaur, P., Lee, R. C. H., Ramphan, S., Kuadkitkan, A., Wikan, N., ... Smith, D. R. (2015). Activity of andrographolide against chikungunya virus infection. Scientific Reports, 5(1). https://doi.org/10.1038/srep14179

Yang, M. H., Ali, Z., Khan, I. A., \& Khan, S. I. (2014). Anti-inflammatory Activity of Constituents Isolated from Terminalia chebula. Natural Product $\begin{array}{lll}\text { Communications, } & 9(7), & 1934578 X 1400900 .\end{array}$ https://doi.org/10.1177/1934578x1400900721 